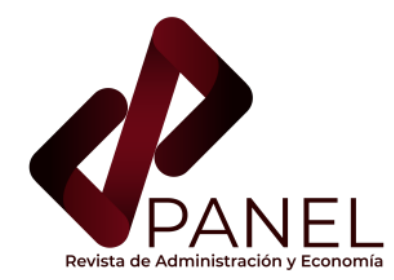

\title{
Balanza de pagos y emisión de bonos soberanos en Bolivia, repercusiones en la tasa de interés
}

\author{
Balance of payments and issuance of sovereign bonds in Bolivia, impacts on \\ the interest rate
}

Artículo recibido en septiembre 2020

Arbitrado en octubre 2020

Aceptado en octubre 2020

Publicado en enero 2021

\section{Rubén Aguilar Cruz \\ raceconomia@yahoo.es \\ ORCID: 0000-0001-7654-9970 \\ Centro Boliviano de Economía \\ Santa Cruz - Bolivia}

Palabras clave

\section{RESUMEN}

ABSTRACT

Keywords
La brecha Ahorro-Inversión en la economía boliviana se tornó negativa en los últimos años. En un entorno de menores entradas de capital, desaceleración del sector real acentuada por los efectos de la pandemia del COVID-19 y una mayor preferencia de los residentes por la moneda extranjera; se atendió las necesidades de liquidez con cargo a reservas internacionales. El estudio buscó evidencia empírica para evaluar el impacto del régimen cambiario actual en un entorno de libre movilidad de capitales sobre la tasa de interés y su posible reacción ante una nueva emisión de deuda soberana. Bajo un enfoque mixto con alcance correlacional y considerando la evolución de las variables en el tiempo, se obtuvieron resultados a favor de los postulados teóricos y se concluyó que, si bien la emisión de bonos soberanos en los mercados internacionales estabilizaría la balanza de pagos, probablemente generaría un nuevo punto de referencia para los rendimientos dentro del país.

Política monetaria; política fiscal; cuenta corriente; tasa de interés; mercados financieros

In recent years, the Savings-Investment gap in the Bolivian economy has turned negative. In an environment of lower capital inflows, a slowdown in the real sector accentuated by COVID-19 and with a greater preference for foreign currency by residents, liquidity charged to international reserves were privided. The study sought empirical evidence to evaluate the impact of the current exchange rate regime with free capital mobility on the interest rate and its possible reaction to the issuance of sovereign debt. Under a mixed approach with correlation scope and considering the evolution of the variables of interest over time, results were obtained in favor of the theoretical postulates and it was concluded that, although the issuance of sovereign bonds in international markets would stabilize the balance of payments, it would probably generate a new benchmark for yields within the country.

Monetary policy; fiscal policy; current account; Interest rate; financial markets 


\section{INTRODUCCIÓN}

Bolivia, es un país ubicado en la parte occidental de América del Sur, cuenta con una población cercana a once millones de habitantes y un Producto Interno Bruto (PIB) por persona al cierre de 2019, de USD 3.591. Al igual que a muchas economías de la región, en las que la explotación de los recursos naturales es una fuente importante de ingresos para el erario nacional, la pandemia ocasionada por la enfermedad por coronavirus (COVID-19) generó importantes ajustes en las cuentas macroeconómicas, principalmente asociadas al vínculo o dependencia de la economía local al resto del mundo.

Al escenario señalado, se le debe sumar que el país atravesaba una situación política delicada porque en octubre de 2019, fruto de varios desacuerdos sociales, se pide la renuncia del Presidente recientemente electo Evo Morales Ayma, quien iba a empezar un nuevo mandato tras mantenerse en el cargo catorce años; esta situación derivó a la constitución de un gobierno transitorio a cargo de la Presidente Jeanine Áñez Chávez, con el objetivo de llamar a una elección en los plazos previstos, hecho que se fue aplazando a causa de las medidas de emergencia sanitaria generadas por la pandemia. Las elecciones generales, se desarrollaron el 18 de octubre $y$, el 8 de noviembre Luís Arce Catacora asumió la presidencia del país.

El entorno político y social descrito implicó en materia económica varios desacuerdos entre el Órgano Ejecutivo y la Asamblea Legislativa Plurinacional de Bolivia, única instancia con la facultad de sancionar y aprobar leyes. La necesidad de contar con la normativa señalada recaía en poder gestionar y recibir recursos desde el exterior con el propósito de fortalecer la balanza de pagos del país.

Para contextualizar, las Reservas Internacionales Netas (RIN) del país, administradas por el Banco Central de Bolivia (BCB) en el marco de la Ley 1670 de 31 de octubre de 1995 y la Constitución Política del Estado, alcanzaron el nivel más alto en noviembre de 2016 (USD 15.477MM, 46,6\% del PIB de entonces); sin embargo, un mes antes de los conflictos sociales llegaron a USD 7.650MM (septiembre de 2019), una reducción aproximada a la mitad del máximo alcanzado en menos de cinco años.

La balanza de pagos daba cuenta que, para mantener el fuerte programa de inversión pública, inmerso en la planificación de largo plazo del país (Agenda Patriótica 2025 y en el Plan Nacional de Desarrollo Económico y Social 2016-2020), era necesario contar con recursos del exterior, dado el déficit en la cuenta corriente de la balanza de pagos, reflejo del escaso ahorro nacional, o de lo contrario la economía para atender estos programas debería continuar usando reservas internacionales.

El Órgano Ejecutivo, en el año 2019, todavía bajo la administración del Presidente Evo Morales Ayma, aprobó en la Ley del Presupuesto 2020 la emisión de USD 1.500MM de bonos soberanos en mercados internacionales, los cuales no pudieron ser concretados en la administración transitoria porque se argumentó la necesidad de la aquiescencia de la Asamblea Legislativa Plurinacional. 
En el contexto descrito, los hogares y las firmas al interior del país continuaron cambiando liquidez en moneda nacional en el sistema financiero por liquidez en moneda extranjera, también incrementaron sus tenencias de moneda extranjera fuera del sistema financiero (circulante en moneda extranjera) y la posición creciente de activos en el resto del mundo por parte de estos, también se fue acentuando. Todos estos acontecimientos observados desde 2018, tuvieron como corolario en 2019 el Reporte de Balanza de Pagos y Posición de Inversión Internacional-2019 del BCB, que el país habría perdido USD 2.479MM, de los cuales el uso de USD 1.618MM era desconocido y estaba reportado como "Errores y Omisiones". Ya en 2020, la gestión se cierra con USD 5.276MM de reservas internacionales.

En la administración gubernamental presente, se promulga la Ley del Presupuesto 2021, que en su Artículo 11 faculta al Órgano Ejecutivo la emisión de USD 3.000MM de bonos soberanos en mercados internacionales.

El estudio tuvo por objetivo estudiar la evidencia empírica para evaluar los postulados de la teoría económica inherentes a repercusiones en la tasa de interés a raíz de un régimen cambiario fijo en entornos de movilidad de capitales y, evaluar la importancia de contar con liquidez externa proveniente de emisión de bonos soberanos. En este contexto, las preguntas que el documento respondió son: ¿Es imperante contar con USD 3.000MM de liquidez internacional?, ¿cuáles serían las repercusiones que internamente implicaría la no emisión de estos títulos de deuda a nivel internacional?, ¿son suficientes estos recursos para estabilizar la balanza de pagos? Contar con resultados que atiendan estas interrogantes, permitirá tener una idea del tamaño de las necesidades de financiamiento que tiene la economía nacional y sus repercusiones en la tasa interna de interés.

\section{METODOLOGÍA}

El estudio se realizó bajo el enfoque mixto con alcance correlacional, sustentado en la teoría económica. Toda vez que los postulados tratados se acompañaron de evidencia empírica y se construyeron variables para validar su coherencia.

La recolección e interpretación de información respondió a un estudio temporal. Prevaleció el aporte cuantitativo, a partir de la recolección, estimación e interpretación de variables macroeconómicas; el componente cualitativo sirvió de apoyo para el análisis del contexto en la interpretación del entorno económico.

En este sentido, se compiló información macroeconómica de fuentes oficiales (Instituto Nacional de Estadística, Ministerio de Economía y Finanzas Públicas, Banco Central de Bolivia, Autoridad de Supervisión del Sistema Financiero) en torno a las variables de interés. A continuación, se detalla la naturaleza de la información para cada relación estudiada.

Brecha Ahorro-Inversión. Para su construcción se tomó información del Ingreso Nacional Bruto Disponible para el periodo 1990-2016, para los siguientes años se realizaron estimaciones de acuerdo con los lineamientos del Sistema de Cuentas Nacionales (2008) y el Sexto Manual de Balanza de Pagos y Posición de Inversión Internacional 
(MBP6), presentado por el Fondo Monetario Internacional (2009). Las cifras correspondientes a la parte fiscal fueron aproximadas mediante las operaciones de financiamiento que están disponibles en la página web del $\mathrm{BCB}$, dado que a la fecha del estudio aún no existían cifras cerradas del ejercicio sobre la línea al 2020. Por complemento, se obtuvo la posición del sector privado.

Balanza de Pagos. Se contó con cifras oficiales hasta el tercer trimestre de 2020, al ser su uso de referencia, no hubo necesidad de realizar estimaciones al cierre de esa gestión. Las cifras de RIN en oro y RIN sin oro se obtuvieron de la página web del BCB y no se realizó ningún tratamiento adicional, más que el distinguir su composición en el total de RIN.

Ingresos tributarios. LoS datos presentados hasta el 2019, corresponden a los registrados en el balance del Sector Público No Financiero (SPNF). Para el cierre de 2020, se realizaron las estimaciones considerando la actividad real en un entorno de baja inflación.

Ingresos por hidrocarburos. Se tomaron los precios del petróleo y las exportaciones de gas natural por mercados, cuya fuente al cierre de 2020 fue el Instituto Nacional de Estadística (INE).

Balance del sector público no financiero. Se contó con datos oficiales hasta el cierre de 2019, para 2020 el ingreso fue estimado según se mencionó en el párrafo anterior. La parte del gasto corriente, al ser inflexible, se mantuvo acorde a la tasa de crecimiento de los últimos años. El gasto de capital se correlacionó uno a uno con las importaciones de bienes de capital disponibles en el INE. El financiamiento pudo monitorearse de manera continua por medio de las operaciones de mercado abierto disponibles en el Ente Emisor.

CIN y RIN. Las cifras que miden la emisión por el lado del origen corresponden a datos oficiales del $\mathrm{BCB}$, así como la descomposición del CIN que está disponible en el balance del BCB. Un hecho similar sucede con la información de la tasa pasiva para depósitos a plazo fijo a 360 días.

La información del IGAE corresponde a la publicación al mes de noviembre de 2020 por el INE, para su uso se comparó esta información con los crecimientos acumulados a similar periodo de las gestiones anteriores.

PFF. Los datos fueron compilados de las metas cuantitativas de los tres PFF de 2020, los cuales están disponibles en el BCB.

Ratio moneda nacional/moneda extrajera. Se tomó información primaria del BCB.

Posición de cambios del sistema bancario. Se calculó considerando los balances del sistema bancario disponibles en la Autoridad de Supervisión del Sistema Financiero.

\section{Rendimientos de los bonos soberanos.} Los datos fueron obtenidos de varias fuentes, cuya publicación primaria es Bloomberg, entre las fuentes consultadas están los estados financieros desagregados del sistema bancario y publicaciones al respecto. 
RESULTADOS Y DISCUSIÓN

\section{La brecha Ahorro Inversión}

La teoría económica da cuenta que un ejercicio de consistencia es poder calcular la brecha Ahorro-Inversión (Agenor, 2004), para este propósito se tomó en cuenta el marco analítico de las cuentas nacionales.

En este sentido, todo el ejercicio parte de la identidad del PIB por el lado de la demanda, a la cual se le agrega el pago neto que reciben los factores internos de producción desde el exterior. Luego, tras algunas operaciones aritméticas, se estable el vínculo donde ahorro nacional menos inversión nacional deben ser iguales al resultado de la cuenta corriente de la balanza de pagos; lo que equivale a decir, si una economía necesita de recursos para solventar su inversión y en el mercado interno los recursos existentes son insuficientes, se debe acudir al ahorro externo (ecuación 1$)^{1}$

$$
\left(S_{t}^{g}-\boldsymbol{I}_{t}^{g}\right)-\left(\boldsymbol{S}_{t}^{p}-\boldsymbol{I}_{t}^{p}\right)=C C
$$

Donde el superíndice refleja la postura del gobierno ( $g$ ), del sector privado $(p)$ y " $S$ " es el ahorro e, "I" es la inversión, "CC" es el resultado de la cuenta corriente de la balanza de pagos.

Con datos observados del PIB y aproximando el Ingreso Primario e Ingreso Secundario del Ingreso Nacional Bruto disponible, con información de las rúbricas de la Balanza de Pagos, se encuentra que la economía boliviana mantiene una brecha negativa desde 2014 (Gráfico 1).

\section{Gráfico 1}

Desagregación de la Brecha Ahorro - Inversión (En miles de MM de BOB)

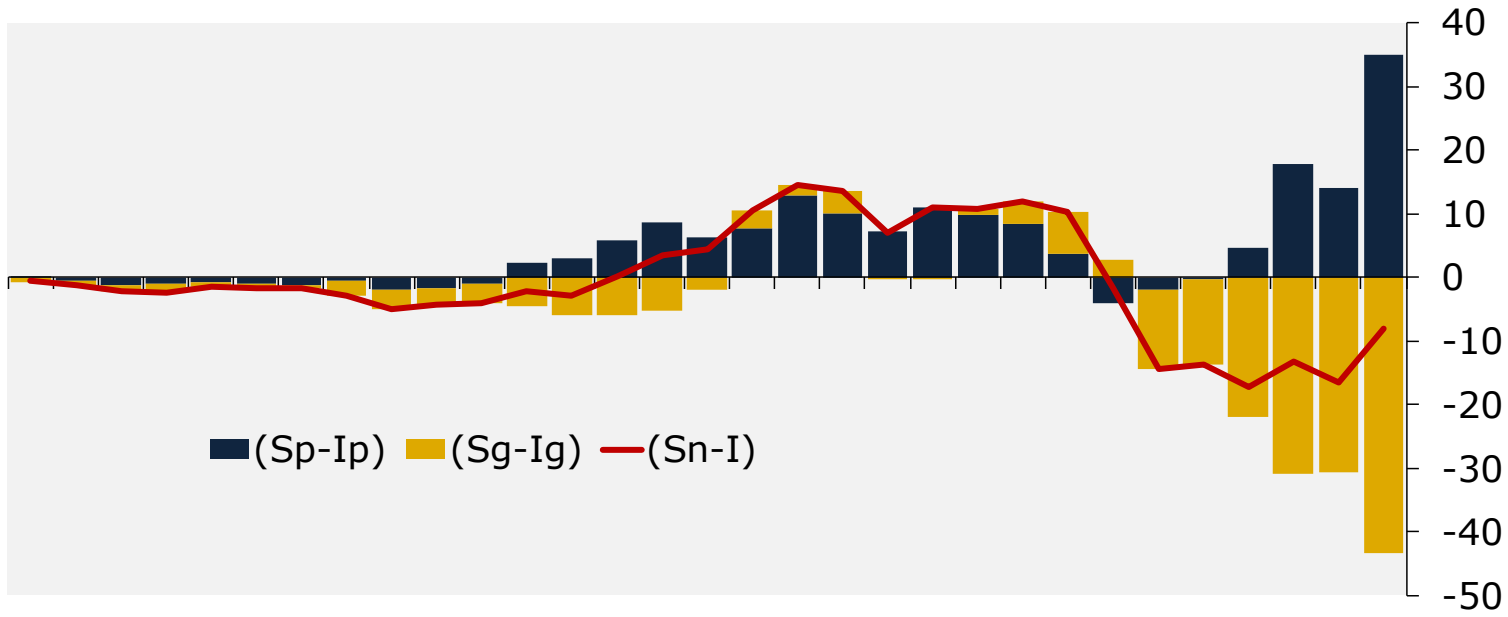

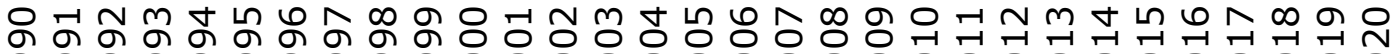

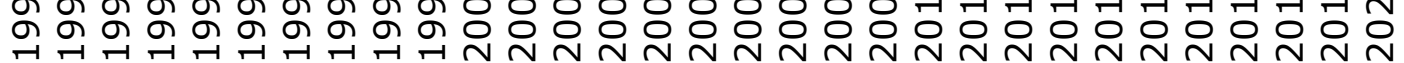

Fuente. Instituto Nacional de Estadística. Desde 2017 estimaciones propias

${ }^{1}$ El desarrollo aritmético se encuentra en Croce, Da Costa y Juan-Ramón (2002). 
El Gráfico 1 revela que la brecha negativa Ahorro-Inversión (S-I) está explicada por la brecha fiscal, en tanto que la brecha privada es positiva; empero, pequeña como para mantener una brecha global con este signo.

\section{Balanza de Pagos}

La Balanza de Pagos junto a la posición de inversión internacional, es la única estadística que muestra el vínculo real y financiero de la economía nacional con el resto del mundo (Tabla 1 ).

Tabla 1

Balanza de Pagos (En MM USD)

\begin{tabular}{|c|c|c|c|c|c|c|c|c|c|c|c|c|}
\hline & \multicolumn{3}{|c|}{2017} & \multicolumn{3}{|c|}{2018} & \multicolumn{3}{|c|}{2019} & \multicolumn{3}{|c|}{2020} \\
\hline & Credito & Debito & Neto & Credito & Debito & Neto & Credito & Debito & Neto & Credito & Debito & Neto \\
\hline Cuenta corriente & 11.393 & 13.291 & -1.898 & 12.077 & 13.900 & -1.823 & 11.827 & 13.175 & -1.348 & 6.146 & 6.517 & -371 \\
\hline Bienes & 8.134 & 8.681 & -547 & 8.895 & 9.354 & -460 & 8.819 & 9.055 & -236 & 4.700 & 4.466 & 234 \\
\hline Servicios & 1.455 & 3.081 & -1.626 & 1.459 & 3.089 & -1.631 & 1.405 & 2.841 & -1.436 & 505 & 1.360 & -855 \\
\hline Ingreso primario & 181 & 1.291 & -1.110 & 181 & 1.157 & -976 & 139 & 953 & -814 & 78 & 552 & -474 \\
\hline Ingreso secundario & 1.623 & 238 & 1.385 & 1.542 & 299 & 1.243 & 1.464 & 326 & 1.138 & 863 & 140 & 723 \\
\hline \multirow[t]{2}{*}{ Cuenta Capital } & 12 & 9 & 3 & 9 & 2 & 7 & 7 & 5 & 2 & 9 & 1 & 8 \\
\hline & Activo & Pasivo & Neto & Activo & Pasivo & Neto & Activo & Pasivo & Neto & Activo & Pasivo & Neto \\
\hline Cuenta financiera & 93 & 2.713 & -2.620 & -1.742 & 1.013 & -2.755 & -1.919 & 1.044 & -2.963 & -600 & -159 & -441 \\
\hline Inversión directa & 80 & 712 & -633 & -84 & 302 & -387 & 48 & -238 & 286 & 237 & -328 & 565 \\
\hline Inversión de cartera & -47 & 1.030 & -1.077 & -777 & 24 & -800 & -522 & 13 & -536 & -383 & -1 & -381 \\
\hline Otra inversión & 293 & 970 & -678 & 348 & 687 & -339 & 1.394 & 1.269 & 125 & 230 & 170 & 60 \\
\hline Activos de reserva & -232 & 0 & -232 & -1.230 & 0 & -1.230 & -2.839 & 0 & -2.839 & -685 & 0 & -685 \\
\hline Errores y Omisiones & & & -725 & & & -939 & 0 & 0 & -1.618 & 0 & 0 & -78 \\
\hline
\end{tabular}

Banco Central de Bolivia, Reporte de Balanza de Pagos y Posición de Inversión Internacional (2020). Las cifras para 2017, 2018 y 2019, son anuales y para 2020 corresponden acumuladas al último trimestre

Como lo muestra la tabla 1 , por rúbricas, la Cuenta Corriente de la balanza de pagos, presenta un continuo déficit explicado por el resultado de Servicios y sobre todo por el retroceso de los ingresos provenientes de las exportaciones de bienes; el Ingreso Primario asociado a la renta de los factores es continuamente negativo debido a la reinversión de utilidades y política de dividendos de la Inversión Directa (ID), cada año en menor magnitud; por su parte, los ingresos provenientes de las remesas se mostraron estables.
La Cuenta Financiera revela que la economía pasa de ser emisora neta de pasivos de ID y receptora de flujos de divisas, a mostrar cifras negativas y salida de recursos del país. La cuenta de Inversión de Cartera revela que de haber recibido más de USD 1.000MM en 2017, al tercer trimestre de 2020 no sólo no habría alojado entrada de recursos, sino también, aunque en una mínima magnitud, se habría revertido. 
Un hecho que no debería ser un patrón es el registro de Errores y Omisiones en los Reportes de Balanza de Pagos por el BCB; desde 2017 hasta el tercer trimestre de 2020, la economía nacional hizo uso de sus RIN por USD 4.986MM, de los cuales el BCB desconoce el destino de USD 3.360MM (en el marco del MBP6, 2009). Esta pérdida de recursos podría estar asociada a un subregistro de las importaciones, o a una acumulación de activos de residentes nacionales en el resto del mundo, o la combinación de ambos.

El periodo de acumulación RIN, o como figura en el MBP6: Activos de Reserva (p.105), se inicia a partir de 2003 hasta alcanzar un máximo de USD 15.477MM, en noviembre de 2014 (46,6\% del PIB); a partir de entonces la economía empieza a hacer uso de estos importantes buffers hasta cerrar la gestión 2020 en USD 5.276MM (Gráfico 2).

\section{Gráfico 2}

RIN, RIN sin Oro y RIN en Oro (En millones de USD)

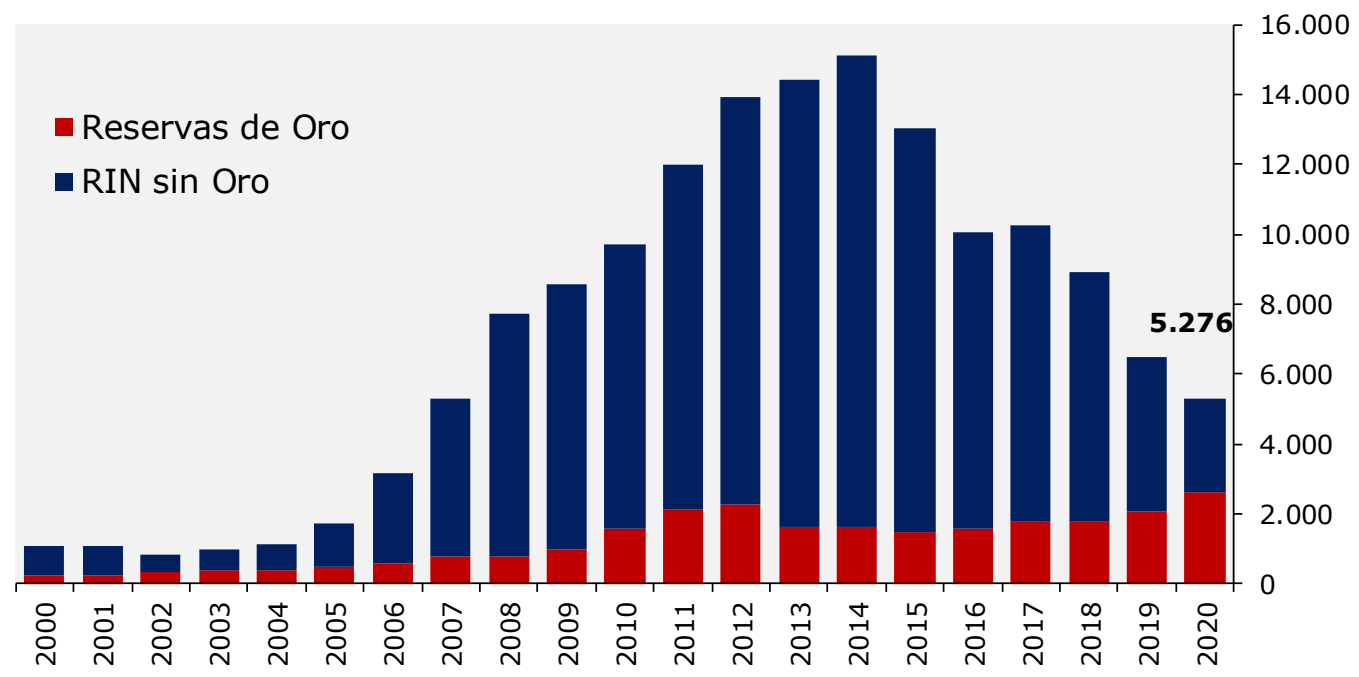

Banco Central de Bolivia, Información Estadística Semanal (2020).

El Gráfico 2, además de mostrar la evolución total de los activos de reserva, muestra la importancia que tienen en la composición los activos en oro, aproximadamente la mitad, al cierre de 2020.

La brecha ahorro-inversión negativa, guarda coherencia con el déficit existente en la cuenta corriente de la balanza de pagos y el inicio de un periodo de uso de RIN. En este sentido, Aguilar (2019) muestra los fundamentos que subyacen a los periodos de tiempo que caracterizaron el dinamismo de las RIN.

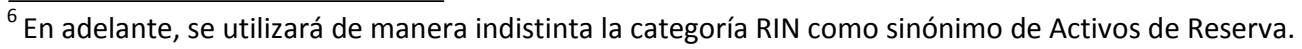


Un elemento que debe ser analizado considerando los instrumentos jurídicos apropiados, es la verdadera disponibilidad de las reservas en oro; si bien estas fueron obteniendo una mayor importancia en la composición de las reservas totales, se debió a la cotización internacional del mineral. En muy pocas ocasiones el BCB incrementó el quantum de sus tenencias en oro y fue, en cantidades mínimas.

Desde el punto de vista del MBP6 (2009) los activos de reserva tienen como función satisfacer las necesidades de financiamiento de la balanza de pagos (P.105), para intervenir en el mercado cambiario y defender la paridad; vale decir, que cuando el Ente Emisor necesite monetizar las reservas en oro, debería convertirlas en activos líquidos de forma inmediata; empero, el artículo 16 de la Ley 1670, da cuenta que en caso de pignoración del oro, esta operación debería contar con aprobación legislativa. Aparentemente, podría existir una restricción normativa que dificulte al $B C B$ a contar con el Oro Monetario en forma de activos líquidos.

\section{La Política Fiscal}

Los ingresos del Sector Público No Financiero (SPNF) se explican principalmente (cerca de cuatro quintas partes) por las recaudaciones tributarias y los ingresos por hidrocarburos, cuya principal fuente es el Impuesto Directo a los Hidrocarburos (IDH). Desde 2014, los ingresos tributarios mostraron una inflexibilidad al alza, en tanto que los provenientes de la renta petrolera mostraron un ajuste importante a la baja, principalmente por un efecto precio de exportación de gas natural $y$ en menor medida por una menor nominación de este energético por parte de Brasil y Argentina, mostrando una importante dependencia que tiene el SPNF al entorno internacional.

La combinación del resultado descrito por encima la línea y la poca flexibilidad del gasto público se tradujo en un incremento de las necesidades de financiamiento del SPNF (Gráfico 3). 


\section{Gráfico 3}

Balance del Sector Público No Financiero (En millones de BOB)

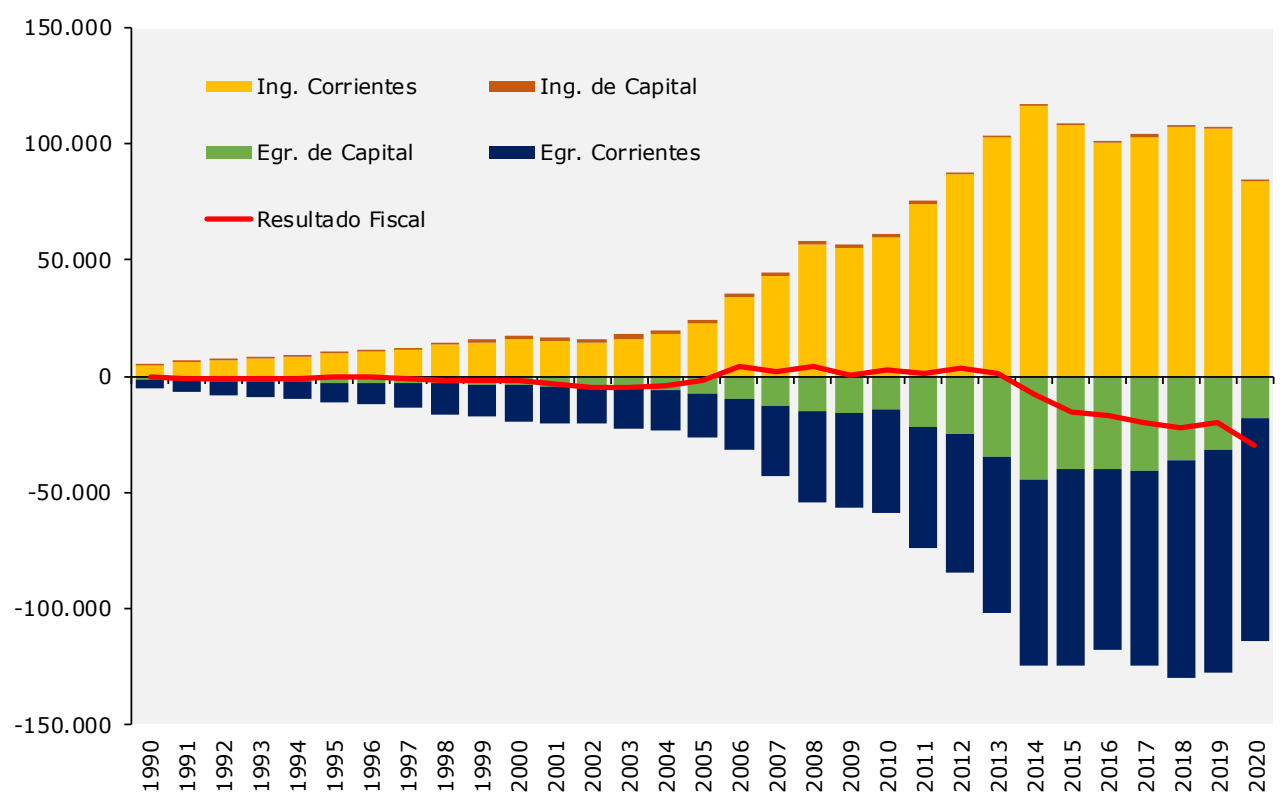

Ministerio de Economía y Finanzas Públicas, Cifras Fiscales (2019). El dato para 2020 es estimación propia

El Gráfico 3, muestra la coherencia del uso de reservas internacionales correlacionada con la política gubernamental de intensificar la inversión pública, asociada en gran medida al gasto de capital. Esta política de dar un mayor protagonismo a la iniciativa gubernamental y que el estado forme parte activa de la economía per se, fue implementada desde 2006 y formó parte de los diferentes planes de desarrollo que marcaron la política económica desde entonces. Por su parte, la administración económica transitoria (2019-2020) redujo la asignación de recursos a estos emprendimientos estatales, porque se consideró que al momento no se tenía el retorno esperado.

\section{Financiamiento con Bonos Soberanos}

El principal precio financiero internacional que daría cuenta de la posición de Bolivia, lo constituye la cotización de los Bonos Soberanos. Bolivia, entre 2012 y 2017 consiguió importantes recursos en mercados internacionales de capitales, emitiendo Bonos con respaldo del Tesoro General de la Nación (Bonos Soberanos), cuyo valor nominal alcanza USD2.000MM. La primera emisión de USD500MM se la realizó en octubre de 2012, con cupón de 4,875\%; la segunda de USD500 MM, en agosto de 2013, con cupón de 5,95\% y la última de USD1.000 MM, en marzo de 2017. La maduración de estos títulos recae en el periodo comprendido entre 2022 y 2028. 
Gran parte de los tenedores de los Bonos Soberanos son instituciones financieras bolivianas, donde estos títulos ocupan niveles muy representativos en el portafolios de sus inversiones. En efecto, con información disponible en los espacios oficiales de las autoridades que regulan los mercados financieros, podría señalarse que cerca de tres cuartas partes del total de las emisiones están inmersas en las hojas de balance de estas instituciones; con la siguiente desagregación: Entidades de Intermediación Financieras (EIF) cerca de USD350 MM, Administradoras de $\begin{array}{llll}\text { Pensiones } & \text { (AFP) USD850 MM }\end{array}$ aproximadamente, los restantes casi USD300 MM en otras compañías y administradoras de fondos.

\section{La Política Monetaria}

En los últimos años, la orientación expansiva de la política monetaria estuvo orientada al financiamiento del Crédito Interno Neto (CIN), principalmente al Sector Público. Destaca que en el entorno del COVID-19, el crecimiento de la emisión monetaria se daba en un contexto de fuerte pérdida de RIN y una expansión del CIN (Gráfico 4).

\section{Gráfico 4}

Emisión por el lado del origen (En MM de BOB)

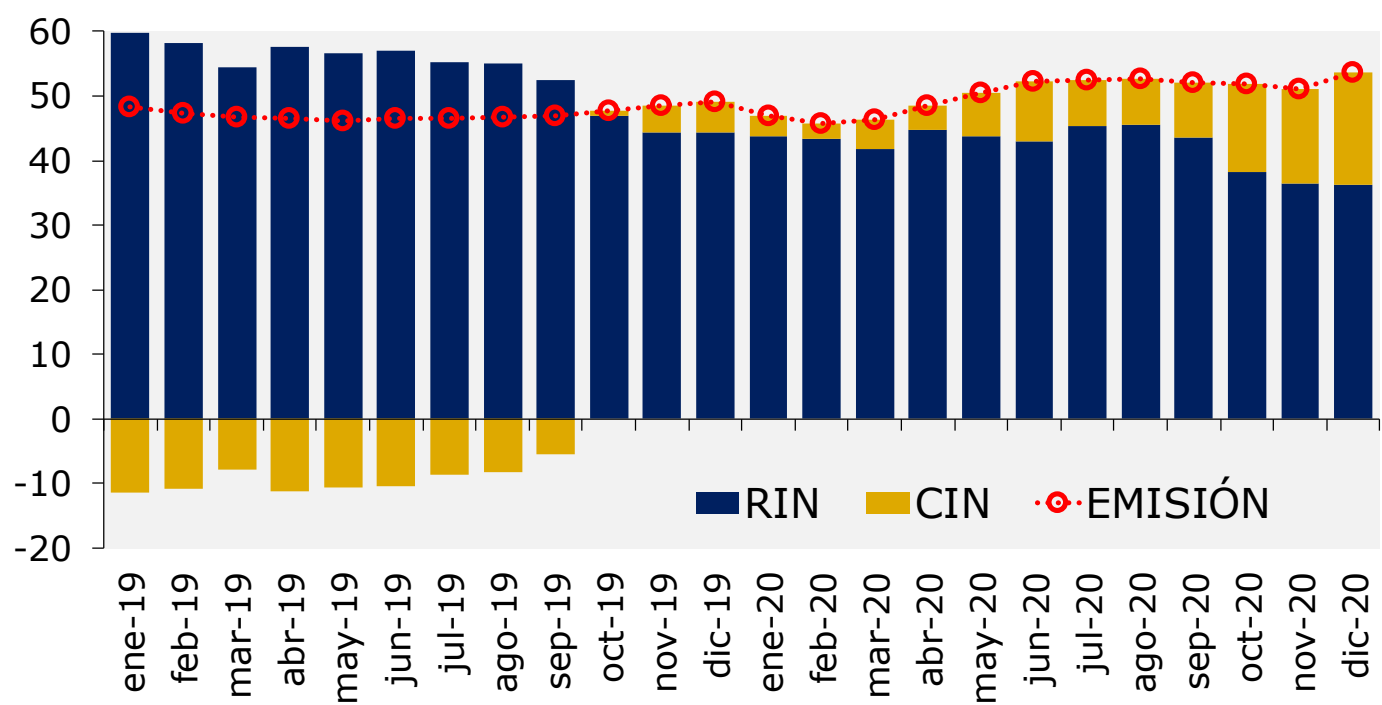

Banco Central de Bolivia, Boletín Mensual º313 (2020) 
El Gráfico 4, permite descomponer el balance del $B C B$ por el lado del activo y se revela que la fuerte expansión del CIN se da por el financiamiento principalmente a empresas del sector público y en menor medida, no por ello menos importante, al gobierno central.

Por su parte, también contablemente el sistema financiero habría recibido una expansión del CIN por parte del $B C B$, el principal impulso se dio el año 2013 con la aprobación del Fondo para la Revolución Productiva (FINPRO) mediante Ley 232 de 9 de abril de 2012; sin embargo, Mediante el Decreto Supremo N N 1367 del 3 de octubre de 2012, se designa al Banco de Desarrollo Productivo (BDP) como la entidad financiera fiduciaria del Fideicomiso del FINPRO encargada de gestionar desembolsos con empresas públicas; así también, con sociedades comerciales con participación mayoritaria del Estado. En stricto sensu, también el CIN al sistema financiero tuvo un último fin, atender las necesidades del estado.

Si bien en 2020 el CIN al sistema financiero fue importante, lo fue en la medida que implicó un cambio de portafolio para las Entidades de Intermediación Financiera (EIF) que mantenían inversiones en forma de encaje en el $B C B$ en moneda extranjera. Con las reformas de encaje, estas inversiones fueron canjeadas por moneda nacional; un hecho similar se da desde fines de
2019, donde las EIF podían hacer operaciones de reporto con el $\mathrm{BCB}$, dejando como resultado colateral inversiones efectuadas en EIF con cierta calificación de riesgo; ambas medidas no implicaron recursos genuinos para las EIF porque tanto las inversiones en encaje en moneda extranjera como el portafolio de inversiones forman parte de sus activos líquidos.

El balance global de las operaciones del BCB da cuenta que en 2020 la emisión monetaria habría alcanzado su máximo nivel histórico, si bien en un entorno de baja inflación la cual no es una variable de preocupación, al menos por el momento; el comportamiento de la actividad real no guarda unísono con el monetario, toda vez que en el último lustro la tasa de crecimiento del PIB mantuvo una tendencia de ajuste a la baja.

La expansión de la emisión monetaria, por la que viene atravesando la economía boliviana, tiene dos particularidades: i) La expansión del dinero se realiza en un entorno donde la actividad real venía desacelerándose en los últimos años y; ii) el fin último de la emisión de dinero es el financiamiento de las cuentas fiscales.

\section{a) Desaceleración de la actividad real. El} gráfico 5, muestra la tasa de crecimiento acumulada del IGAE, al mes de noviembre de cada gestión. 


\section{Gráfico 5.}

Tasa de crecimiento acumulada del IGAE (En porcentaje)

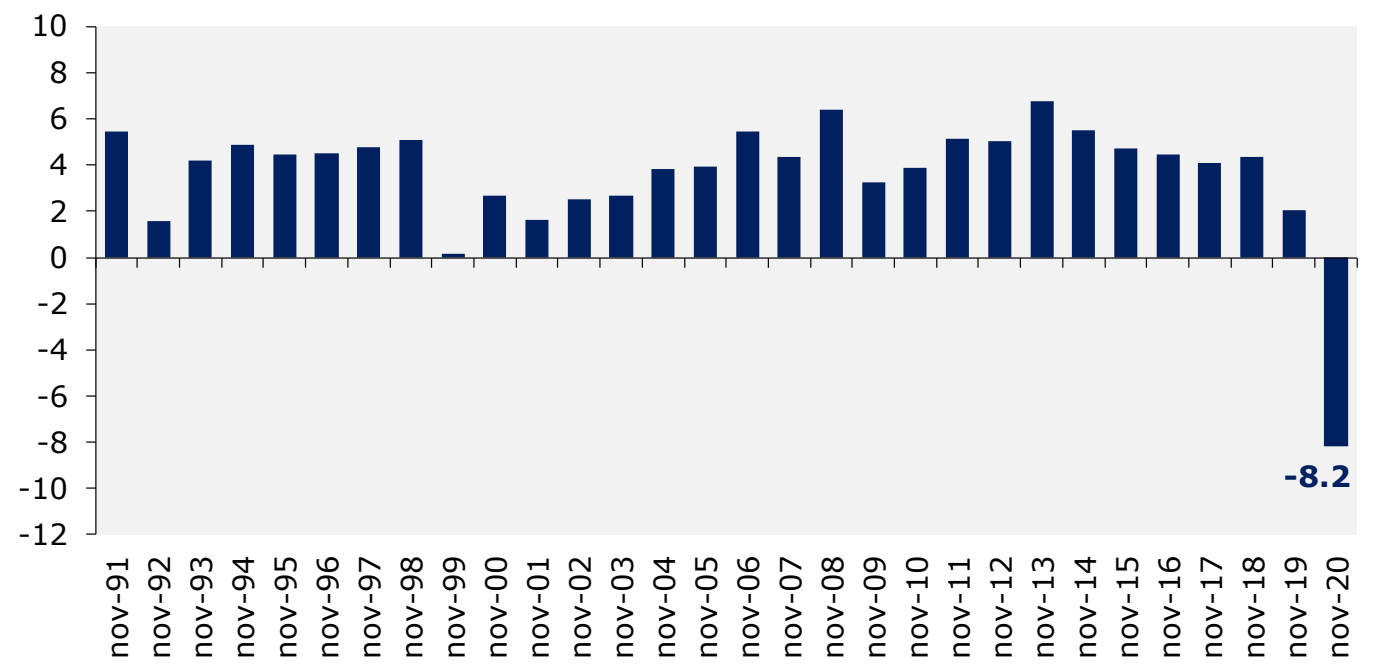

Instituto Nacional de Estadística

El Gráfico 5, permite observar una importante desaceleración de la actividad real de la economía boliviana; en efecto, luego de haber alcanzado un máximo de $6,8 \%$ en 2013 , este guarismo vino contrayéndose hasta alcanzar un 2,1\% en 2019 y pasar a una cifra negativa asociado a la pandemia en 2020. b) Financiamiento del déficit fiscal. La tabla 2, muestra las cifras inmersas en el Programa Fiscal Financiero (PFF), que es el único documento macroeconómico en el país, que fija metas explícitas para variables, monetarias, financieras y fiscales.

\section{Tabla 2}

Programa Fiscal Financiero 2020

6 de febrero 14 de septiembre 7 de diciembre

En millones de bolivianos

\begin{tabular}{lccc} 
Déficit del Sector Público & 19,679 & 32,121 & 32,402 \\
Financiamiento Interno al Sector Público & 6,302 & 12,179 & 24,734 \\
Crédito Interno Neto del BCB al SPNF & 4,137 & 15,553 & 21,435 \\
Crédito Interno Neto total & 8,217 & 15,070 & 12,855 \\
& \multicolumn{2}{c}{ En millones de dólares americanos } \\
RIN & $-1,042$ & $-1,399$ & $-1,367$ \\
\hline
\end{tabular}

Banco Central de Bolivia 
La Tabla 2, además de registrar que en 2020 se firmaron tres PFF, muestra que, en diciembre, la nueva administración gubernamental develaba la necesidad de profundizar aún más el financiamiento del $B C B$ al Ministerio de Economía y Finanzas Públicas (MEFP). El 6 de febrero, en el primer PFF de 2020, el BCB se comprometía a otorgar un financiamiento máximo, con cargo a emisión monetaria de $\mathrm{BOB}$ 4.137MM; fruto de la pandemia el financiamiento fue revisado a BOB 15.553MM y faltando 24 días para cerrar la gestión el financiamiento a ser otorgado llegó a BOB 21.435MM.

La literatura económica, da cuenta que es natural observar un crecimiento de la emisión si esta obedece a sus fundamentos. Barro y Gordon (1983) demuestran que lo complicado para el sistema financiero y para la economía en general se da cuando el banco central introduce algunas sorpresas monetarias, vale decir, cuando el dinero está creciendo por encima de sus fundamentos.

Trabajos más recientes que buscaron evidencia empírica sobre los fundamentos de la emisión, sostienen que estos recaen en la activad real y los objetivos de inflación; Noriega, RamosFrancia y Rodríguez Pérez (2011), encuentran resultados a favor de este postulado en el entorno de calcular un nivel de inflación que maximiza el señoreaje para México; por su parte también existe evidencia para Brasil, Marruecos, Nueva Zelanda y varias economías, de la preponderancia en el corto y largo plazo del nivel de ingreso como fundamental de la emisión (Hamiani, 2018).

Para el caso boliviano, una expansión del dinero en un entorno de desaceleración de la actividad real, ex ante a la pandemia y acentuada mucho más por esta, cuyo principal y único destino sea el financiar el déficit fiscal podría tener repercusiones adversas para la economía global. El destino de la emisión exclusiva para financiar el déficit fiscal se sustenta en que el crédito al sistema financiero del BCB se torna negativo por la acumulación de depósitos en el Ente Emisor, tal como lo muestra el Crédito Interno Neto total del BCB en la tabla 2.

Ahora bien, la teoría económica sostiene que los bancos centrales tienen la posibilidad de expandir la oferta monetaria infinitamente; sin embargo, este accionar podría tornarse peligroso cuanto esa política genera desconfianza en los hogares, las firmas y el sistema financiero, toda vez que la demanda de dinero, de saldos reales, es una demanda por "confianza" y en la medida que ese dinero se traduzca en sustitución de moneda, como lo señalan Calvo y Végh (1992) sería una situación de que la economía local no es una economía estable. En consecuencia, en un entorno de expansión monetaria como la registrado en la economía nacional en los últimos años, el problema podría reflejarse en que los agentes económicos empiecen a sustituir dinero local por moneda extranjera.

Considerando en el numerador del ratio $\frac{M n}{M e}$ los billetes y monedas en poder del público más los depósitos en cuenta corriente en moneda nacional y en el denominador los depósitos vista, caja de ahorro y DPF en moneda extranjera, se encuentra evidencia, de que el indicador alcanza un punto de inflexión en 2018, tras un continuo crecimiento (Gráfico 6). 


\section{Gráfico 6}

Relación moneda nacional, moneda extranjera (en número de veces)

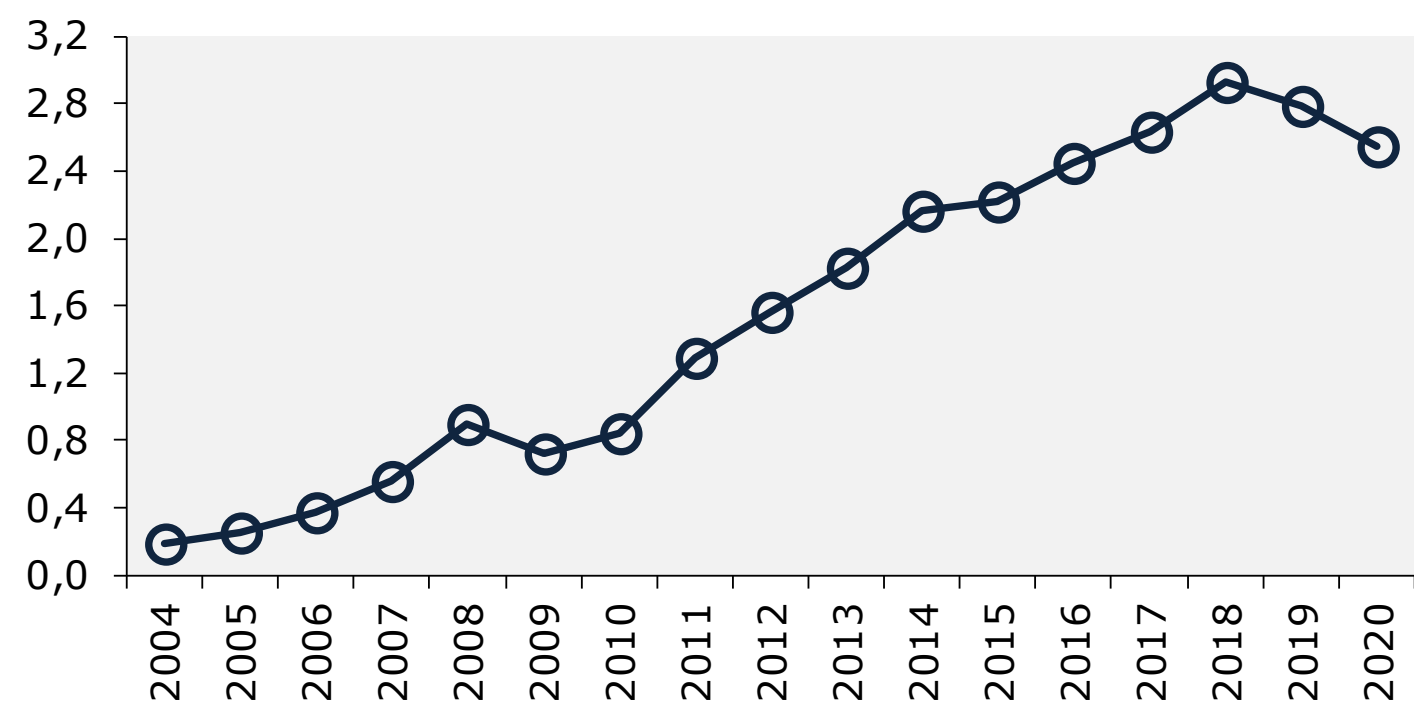

Banco Central de Bolivia, Boletín Mensual №313 (2020)

El comportamiento observado en el gráfico 6, podría ser un indicador de que los hogares y las firmas están empezando a preferir tener menos liquidez en moneda nacional y más en moneda extranjera.

Buscando evidencia en el sistema bancario, para corroborar la preferencia de monedas en este sector, en el gráfico 7 , se observa la evolución de la posición de cambios, entendida como la diferencia entre activos y pasivos en moneda extranjera (en el marco de la Ley 1670, el $B C B$ define el reglamento que regirá estas operaciones para el sistema financiero, para 2020 en adelante, mediante RD011/2019 el directorio del $B C B$, se aprobó una posición simétrica de hasta $50 \%$ del patrimonio contable). 


\section{Gráfico 7}

Posición de Cambios del Sistema Bancario (En número de veces)

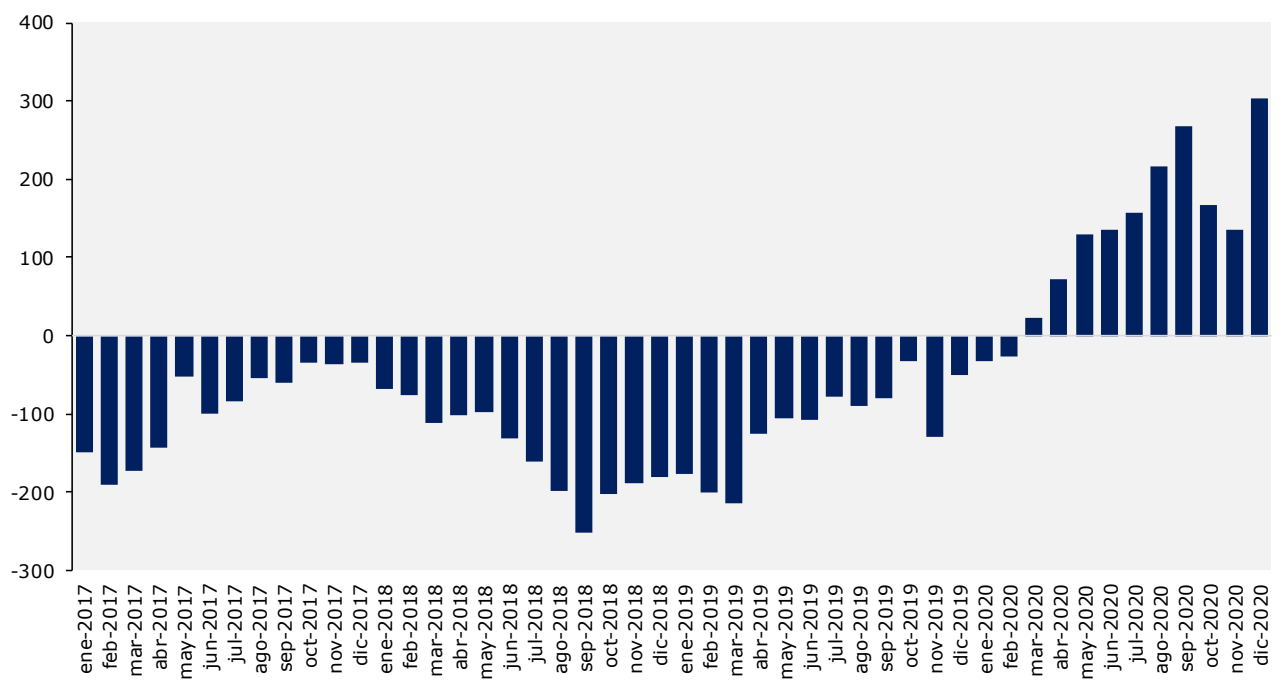

Autoridad de Supervisión del Sistema Financiero, Boletines Estadísticos (2020)

En el Gráfico 7 se observa que en los últimos años fue un patrón relativamente común que el sistema financiero se encuentre en una posición corta o sobrevendida. Esto podría ocurrir por varias razones, la más relevante se da cuando el sistema puede captar ahorros del público en ME y vender esa posición a un tipo de cambio por encima del que lo podrá adquirir del $\mathrm{BCB}$; en línea con Hellerstein y Rain (2011), la preferencia por la moneda foránea podría ser sinónimo de inestabilidad macroeconómica.

Pasar de una posición corta a una larga puede ser una señal muy fuerte sobre la credibilidad del régimen cambiario. Dado que aun pudiendo el sistema financiero encontrar ingresos por operaciones de cambio en un tramo sobrevendido, mantiene una posición larga, pese a que las autoridades del país aseguran que el tipo de cambio no se moverá.

Como se señaló al inicio de esta sección, la expansión del dinero siempre debería analizarse en el contexto por el cual se desempeña la economía; dada esa premisa, la literatura económica admite cierto consenso en creer que las sorpresas monetarias podrían influir "temporalmente" en el producto y empleo, asumiendo la existencia de una curva de Phillips en el corto plazo; sin embargo, esta regla no siempre podría ser válida en el tiempo, creer que una excesiva cantidad de dinero en la economía pueda tener efectos favorables en la actividad real, podría llevar a las autoridades económicas a caer en un problema de "inconsistencia temporal de la política monetaria", labor que laureó a Kydland y Prescott el año 2004 con el Nobel en economía.

Respecto al comportamiento de la sustitución de monedas, preferencia por la moneda extranjera y expectativas por la posición cambiaria; también la literatura empírica ofrece varias regularidades al respecto, una cercana es abordada por Kiguel (2015), que encuentra evidencia robusta analizando siete crisis de balanza de pagos, tres 
crisis bancarias, dos crisis de deuda y seis periodos marcados de alta inflación en Argentina en el periodo de 1952 al 2002, que le lleva a concluir que el retraso cambiario, bajo la creencia de que en el corto plazo se podría estimular el nivel de consumo y la actividad, no es una estrategia sostenida, que además de implicar costos en la sociedad, entendida como el bien mayor, podría implicar fuertes ajustes en las planillas de las oficinas públicas.

Por todo lo expuesto: i) fuerte expansión monetaria en los últimos años; ii) desaceleración de la actividad real en el último lustro; iii) financiamiento del déficit fiscal mediante emisión monetaria; iv) reversión de algunos indicadores de preferencia por la moneda nacional; v) posición de cambios del sistema bancario en situación sobrecomprada o larga, sinónimo de expectativas de movimientos cambiarios; es útil conocer la repercusión de la conjunción de todos estos elementos sobre el comportamiento de las tasas de interés.

Con información temporal desde 1990 al cierre de 2000, se encuentra evidencia de una relación inversa entre la brecha de AhorroInversión y la tasa de interés (Gráfico 8).

\section{Gráfico 8}

Brecha Ahorro - Inversión y Tasas de Interés (En porcentaje del PIB y en porcentaje)

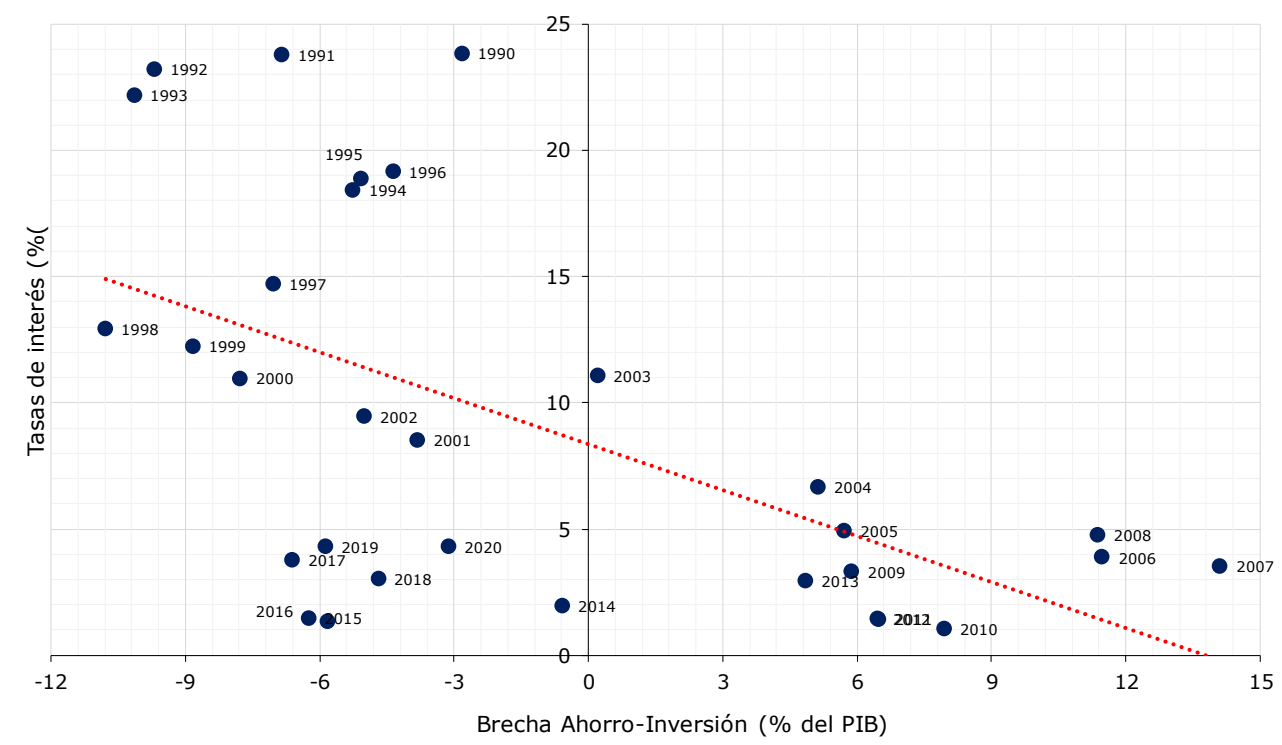

Banco Central de Bolivia; Instituto Nacional de Estadística. Las tasas de interés, corresponden a las operaciones pasivas a plazo fijo a un año. 
Como se observa en el gráfico 8 , incluso en los periodos donde se tenía una brecha positiva (2003 al 2008), la tasa de interés a un año permanecía en niveles relativamente altos (alrededor del 5\% o más); en periodos de brecha negativa (1990-2002) la misma tasa de interés a un año, superó el umbral del 10\%. Lo que está sucediendo en los últimos años (2014 al 2020), la brecha negativa está asociada a una subida importante en las tasas de interés a un año (muy cercanas al 5\%), que es totalmente contraria al observado en los años 2009 al 2013.

La existencia de un déficit en Cuenta Corriente de la balanza es sinónimo del insuficiente Ahorro Nacional para enfrentar los Programas de Inversión. Lo que hace necesario acudir al ahorro externo, que en Bolivia se dio con el uso de activos de reserva, dado el casi nulo aporte de las otras rúbricas de la cuenta financiera de la balanza de pagos.

Bajo este entendido, es muy prudente pensar en fortalecer los activos de reserva, y es así como la Ley del PGE 2021, en el artículo $11^{\circ}$, autoriza al Órgano Ejecutivo la emisión de USD 3.000MM de bonos soberanos en mercados internacionales.

A la fecha los precios de los bonos con vencimiento en el año 2022 y 2023, cotizan alrededor de "la par", esto significa que el tenedor final de este activo, siente conformidad con el rendimiento implícito en cada cupón, acorde a los postulado por Lintner (1965). Un hecho contrario sucede con los títulos con vencimiento en el año 2028, el precio se sitúa muy por debajo del precio del de su emisión y esto se refleja en que el inversionista, para tener estos títulos espera un rendimiento muy por encima del cupón que este ofrece $(4,5 \%)$, las cifras revelan que durante 2020 el mercado esperaba un rendimiento máximo cercano al 9\%, cerrando la gestión cerca al 5,5\%, las marcaciones en los precios reflejadas en los rendimientos son sinónimo de la exigencia que el mercado pide para mantener posición de estos bonos; una señal poco favorable para la nueva emisión de USD 3.000MM, porque se constituyen en un referente que puede generar condiciones indicativas.

Como se mencionó anteriormente, gran parte de los tenedores de emisiones son residentes nacionales y poseen una participación en una nueva emisión tiene importantes repercusiones en sus hojas de balance: i) son activos en dólares, en consecuencia, nominalmente existe una cobertura natural a movimientos cambiarios; ii) la ponderación de estos títulos es nula cuando se computa el Coeficiente de Adecuación Patrimonial (CAP) Financiero, dando holgura a poder expandir la cartera o realizar operaciones contingentes; iii) obtener un cupón 4,5\% a vencimiento (siete años) más una prima por la adquisición a condiciones de mercado, marca una expectativa en el rendimiento de la nueva emisión (la cual con seguridad debería ser mucha más alta que las registradas en 2020 por la emisión con vencimiento al 2028); acorde a lo señalado por la teoría económica de la microeconomía bancaria, que da cuenta que los inversores estarían dispuestos a mantener/abandonar posiciones en bonos, con base a cambios en los rendimientos, los cuales tienen una correlación inversa con el precio de los activos, (Sharpe, 1964), (Linter, 1965) y (Markowitz, 1952).

Ahora bien, dejar la posibilidad de que residentes locales adquieran estos títulos en los mercados internacionales, neutralizan el efecto 
favorable que esta emisión podría tener en la balanza de pagos. Por otra parte, restringir la tenencia a residentes, genera al menos dos efectos: i) los títulos a ser emitidos no son activos muy líquidos en mercados internacionales, vale decir que la emisión de los USD 3.000MM no necesariamente garantiza que los mercados compren esa emisión y; ii) para garantizar un aforo pleno de la emisión, probablemente el cupón tenga que ser demasiado atractivo, lo cual es adverso para el balance del SPNF y marcaría un benchmark nuevo de tasas en el mercado interno, dado que las activas vigentes para segmentos productivos de tamaño grande $y$ mediano son reguladas y llegan al 6\% (Decreto Supremos N²055 de 9 de julio de 2014).

\section{CONCLUSIONES}

El trabajo hizo una revisión de la situación macroeconómica de la economía boliviana, se enfatizó en el comportamiento de las RIN y el fuerte uso que la economía hizo de estas durante los últimos cinco años, en un contexto social, político y de salud adverso por el que se atravesó. Se encontró evidencia de que la política gubernamental de impulsar la inversión pública, se correlaciona con un escenario donde el ahorro nacional no es suficiente como para financiar estas iniciativas y en el entorno de menor flujo de capital al país, el BCB tuvo que atender estos requerimientos usando RIN para así mantener invariante el tipo de cambio.

Asimismo, el trabajo dio cuenta que los hogares y firmas al interior del país, habrían empezado a prestar más atención en recomponer su demanda de dinero, incluyendo un porcentaje más significativo de moneda extranjera en las mismas; este hecho se reforzaba cuando se veía la posición de cambios del sistema financiero que habitualmente se encontraba corto y durante todo el 2020 se encontró en una posición larga.

Una de las preguntas que se buscó responder fue: ¿es imperante contar con los USD 3.000MM de liquidez internacional?, la respuesta va a depender de las necesidades inmediatas que la economía pueda tener para hacer uso de reservas internacionales. Si el plan de gobierno a ser implementado tiene un fuerte componente importado en bienes de capital y productos intermedios, probablemente la demanda de dólares aumente y si, en este entorno, las expectativas por parte de los hogares y firmas son contrarias a la estabilidad del régimen cambiario, debería de realizarse la emisión de los USD 3.000MM lo antes posible, esto podría ser un fuerte escudo para evitar cualquier ataque especulativo a la moneda.

Una segunda interrogante que se planteó fue: ¿cuáles serían las repercusiones que internamente implicarían la no emisión de estos títulos de deuda a nivel internacional? Nuevamente, la respuesta va a depender si es que los residentes locales van a poder adquirir los títulos en los mercados internacionales. Si en caso se restringe la participación, la probabilidad de que se tenga una emisión exitosa es baja y en consecuencia la política gubernamental mantendrá una fuerte necesidad de fondeo de recursos, esto podría impactar en las tasas de interés al alza. En caso de que residentes puedan comprar esos títulos, en algún porcentaje, también habrá un nuevo nivel de tasas de referencia, dado que se compararía un título en moneda extranjera libre de riesgo cambiario y sin ponderación en el CAP del sistema bancario 
versus, la colocación de cartera productiva regulada en moneda nacional a una tasa de interés que probablemente se mantenga en $6 \%$ con una importante ponderación en desmedro del CAP.

Finalmente, una tercera pregunta planteada daba cuenta de si ¿son suficientes estos recursos para estabilizar la balanza de pagos? La respuesta recae en que a la fecha si monetizar las reservas de oro, vale decir que estas se vuelvan divisas, no tienen impedimentos legales, la economía nacional sí estaría contando con la disponibilidad de recursos, de lo contrario, se deberían descontar más de USD 2.500MM de los USD 5.276MM que actualmente figuran como RIN.

\section{REFERENCIAS}

Aguilar, R. (2019). Evaluación de la Economía y del Desarrollo en Bolivia. Cap. 3: "Desempeño del Sector Externo". Konrad Adenauer Stiftung

Autoridad de Supervisión del Sistema Financiero, Boletines Estadísticos (2020). https://www.asfi.gob.bo/index.php/bancosmultiples-boletines.html

Banco Central de Bolivia, Reporte de Balanza de Pagos y Posición de Inversión Internacional (2020)

Banco Central de Bolivia, Boletín Mensual N³13 (2020)

Barro, R. y Gordon D. B. (1983). "A positive Theory of Monetary Policy in a Natural Rate Model". Journal of Political Economy, vol. 91, N4, August

Calvo, G. y Végh, C. (1992). "Currency Substitution in Developing Countries: An Introduction". IMF, Working Paper N92/40
Fondo Monetario Internacional (2009). "Manual de Balanza de Pagos y Posición de Inversión Internacional, sexta versión". IMF

Hamiani, M. (2018). "Monetary Policy and Model of Cyrrency Demand". International Monetary Fund. IMF Working Papers $\mathrm{N}^{\circ} 18 / 28$

Kiguel, M. (2015). "Las crisis económicas argentinas: Una historia de ajustes y desajustes". Grupo Editorial Argentina

Kydland, F. y Prescott, E. (1977). "Rules Rather thah Discretion: The Inconsistency of Optimal Plans". Journal of Political Economy, vol. 85, $\mathrm{N}^{\circ} 3$

Hellerstein, R. y William, R. (2011). "Cash Dollars Abroad," Federal Reserve Bank of New York, Staff Report No. 400, February

Lintner, J. (1965). "Security prices, risk, and maximal gains from diversification". The Journal of FINANCE, vol. XX, Nº4, December

Ministerio de Economía y Finanzas Públicas. (2019).

https://www.economiayfinanzas.gob.bo/vice ministerio-de-tesoro-y-creditopublico.html?id=1786\&articulo=1969\#enfoca $r$

Noriega, M. y Rodríguez, P. (2011), "Uso de agregados monetarios como indicadores de la evolución futura de los precios al consumidor: crecimiento monetario y meta de inflación". Fondo de Cultura Económica; El Trimestre Económico vol.LXXXIV, núm.333, enero-marzo, 2017, pp.5-70

Sharpe W. (1964). "Capital Asset Price: A theory of market equilibrium under conditions of risk". The Journal of FINANCE, vol. XIX, $\mathrm{N}^{\circ} 3$, September 